\title{
Measles outbreaks threaten those averse to vaccine
}

$\mathrm{S}$ even confirmed cases of measles in Toronto, Ontario, so far this year, for a total of 12 in Canada to date, are prompting public health officials to remind doctors that the once-common childhood illness risks establishing a foothold again.

"It's obviously circulating," says Dr. Vinita Dubey, associate medical officer of health with Toronto Public Health. "If we have vulnerable pockets of people in the population, they're at risk of infection."

Dubey is concerned both about the number of cases already reported this year - on average, Toronto sees five cases in an entire year - and the fact that five of the children were unvaccinated and had no history of travel outside of the city.

If vaccination rates for measles drop by just $10 \%$, Toronto could expect large outbreaks of up to 10000 cases in as few as five years, Toronto Public Health estimates. There's now a bigger risk of infection coming from travellers to and from Europe, Dubey says, because of the rise in measles outbreaks in England, France and Spain.
In one of the other cases, a 31-yearold man contracted measles while in Mexico, likely from a British traveller at the same resort, where a New Brunswicker also contracted the same strain of measles. The other Toronto case was a young child who fell ill in January, and whose strain of measles matched one circulating in Quebec and France at the time.

Although measles was once thought near eradication in the Americas and other developed countries because of mass vaccination programs introduced in the 1970s, the disease has been popping up in recent years. In large part, public health officials attribute the resurgence to parental concerns about a since-disproven link between the measles, mumps and rubella (MMR) vaccination, recommended to children as toddlers, and autism.

That link was based on an article Dr. Andrew Wakefield published in 1998 in The Lancet alleging a connection between the vaccine and autism and bowel disease. His research was subsequently proven to be fraudulent and The Lancet later withdrew the paper. But it had received widespread publicity, and the link was made in the public's mind between autism and vaccinations.

Measles is still a leading cause of children's deaths around the world largely preventable by the MMR vaccine, which when administered in two doses protects people $95 \%$ of the time. In 2011, the World Health Organization (WHO) reported that measles caused 158000 deaths, and there are large, ongoing outbreaks in Africa, Asia and Europe. Measles affects more than 1 million people annually around the world, according to the Public Health Agency of Canada.

An outbreak in Wales this year has already affected more than 800 people, 77 of whom required hospitalization. In the United Kingdom, 2- to 18-year-olds are most at risk for measles because $13.6 \%$ of them either did not get vaccinated at all or received only one of the two recommended inoculations.

"There was a big group ... of kids [in the UK] that were not vaccinated, and now measles is spreading like wildfire," says Dubey.

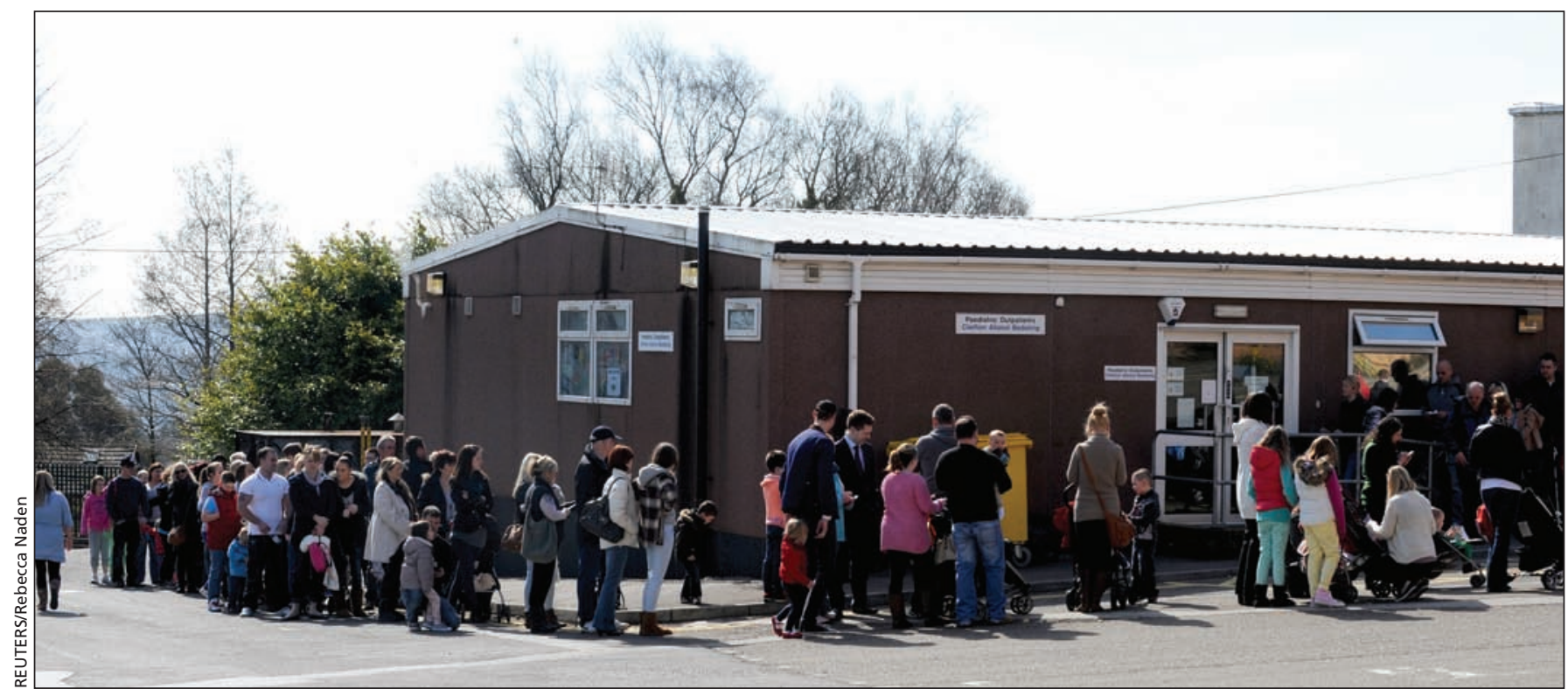

Parents and children line up outside a hospital in south Wales, United Kingdom, to receive vaccinations following an increase of cases of measles in the area. 
In 2011, there was a large outbreak in Canada of 725 cases in Quebec. There were 38 cases of measles in Canada per year on average between 1998 and 2005, and 58 cases per year on average between 2006 and 2010 . The numbers tend to vary substantially from year to year. In 2005, for instance, only six cases were reported. Two years later, the number jumped to 102 but, two years after that, fell back to 14 .

There have been three substantial outbreaks in Canada in recent years 96 cases in Quebec in 2007, 53 cases in Ontario in 2008 and 82 cases in British Columbia in 2010, according to the Public Health Agency of Canada.
Europe also suffered a large measles outbreak in 2011, with more than 30000 reported cases.

Because measles has been so rare in Canada and the United States for decades, family doctors may not immediately recognize it. Symptoms include runny nose, drowsiness, irritability, sensitivity to light, red eyes, fever, small grey/white spots in the mouth and throat, and a blotchy red rash on the face and body. Complications include diarrhea, pneumonia and infections of the brain, with two to three cases per 1000 resulting in death in the developed world.

"Very few young parents and young doctors have ever seen measles, ever, and may not realize how serious it can be," says Dr. Jane Seward, deputy director of the Division of Viral Diseases at the (US) Centers for Disease Control and Prevention.

Measles is a highly infectious disease. People who forgo vaccines and are exposed to the virus have a $90 \%$ chance of acquiring the infection, Dubey says. It spreads through droplets in the air from coughing or sneezing, and has an incubation period of around 10 days, during which symptoms are not visible. - Cullen Bird, CMAJ

CMAJ 2013. DOI:10.1503/cmaj.109-4480 